\title{
RAPID VARIATION OF LOD AND SOLAR FLARES
}

\author{
Chen Li Li Zhian Zhou Zhengfeng \\ Dept. of Astronomy, \\ Beijing Normal University \\ 100875 \\ Beijing, China
}

\begin{abstract}
The sequence of LOD in the period from 1988 to 1991 (which was published in Bulletin B by IERS ) is analyzed. Using the method of multiple filter, all the periodic terms are removed. Therefore, the rapid and outstanding variations of LOD that greater than $0.1 \mathrm{~ms}$ in May, 1989 and Jan., 1990 are shown in the residual series. Because of many bursts of solar flares in the period of Mar.8 to 16 and Nov.7 to 17, there are geomagnetic storms in Mar. and Nov.1989. The strong geomagnetic variation probably induces the rapid variation of LOD.
\end{abstract}

\section{Introduction}

Since 1988, the International Earth Rotation Service (IERS) has comprehensively processed the observation data of VLBI, SLR and LLR. The publishing earth orientation parameters (EOP) were so accurate that in which $\mathrm{x}$ and $\mathrm{y}$ are more precise than $0^{\prime \prime} .001$. And the error of UT is less then $0.1 \mathrm{~ms}$.

It is known that the variation of LOD (length of day) includes long period variation, seasonal variation and high frequency variation. Moreover, it has some irregular rapid variation. Although the order of its magnitude is small, the high processional LOD information given by IERS provides us a sound basis for further study on rapid variation of the earth rotation rate.

In this paper, we use the method of multiple filter to remove various period terms from the LOD series and to keep all unusual high frequency variation. We also discuss the reason of the earth rotation from the angles of AAM (Atmospheric Angular Momentum) and solar flares.

\section{Data and Processing Method}

Data in the paper include: Variation of the earth rotation (LOD), Atmospheric Angular Momentum (AAM), Solar flares (SF) and the index of Geomagnetism (AP). Their sources list in Table 1. 
Table 1. Data sheet

\begin{tabular}{|c|c|c|}
\hline Series & Time interval & Source \\
\hline LOD & $1988-1991$ & IERS Monthly Bulletin B \\
\hline AAM & $1988-1990$ & $\begin{array}{c}\text { European Center for Medium-range } \\
\text { Weather Forecasting (ECMWF) }\end{array}$ \\
\hline SF & $1988-1990$ & Solar Geophysics Data \\
\hline Ap & $1988-1990$ & Solar Geophysics Data \\
\hline
\end{tabular}

To analyze the abnormal rapid variation, we first chose a simulated data series . The steps are as following:

\subsection{Simulated data}

$$
y(i)=0.3 \sum_{i=1}^{9} \sin \left(2 \pi t_{i} / 3 j+\varphi_{i}\right) \quad t_{i}=i=1, \ldots, 1095
$$

In the intervals of $i=50-60,200-230,450-470$ and 800-820 we add some irregularly abnormal values, which magnitude is about 0.6 . Then the simulated data has both period terms and irregularly Then the simulated data has both period terms and irregularly abnormal variations. The curve is shown at the bottom of Figure 1.

\subsection{Multiple filter of simulated data}

In terms of multiple filter theory put forward by Zheng Dawei [1], the frequency response curve is

$$
R=C\left[1-A(f, \varepsilon)^{N}\right]^{M}
$$

where $C$ is a real constant, $N$ and $M$ are positive integers, $A(f, \varepsilon)$ is a response function of Vondrak-Filter. $A(f, \varepsilon)=\varepsilon /\left[\varepsilon+(2 \pi f)^{6}\right]$ and $\varepsilon$ is a smoothing factor. By practice, taking $\varepsilon=0.7, \mathrm{~N}=7, \mathrm{M}=11$, we may filter out all the period terms that over $5 \mathrm{~d}$. The result is shown by the middle curve in Figure 1.

\subsection{Autoregressive (AR) spectral analysis}


Using the method of autoregressive (AR) spectral analysis, we find that, after multiple filtering, only $3 \mathrm{~d}$ period term retains in the series. So we deduct it by the way of period curve fitting. Now we get the residual series (the upmost curve in Fig.1). Obviously, there are only irregular variations in the series.

We process the data of LOD and AAM in the same way. The results are shown in figure 2 and figure 3 .

\section{Analysis}

In figure 2, the bottom curve is LOD series. After multiple filtering, we get the top residual curve which has a few outstanding irregular variations. We know that the average variance of the curve is $0.023 \mathrm{~ms}$. But during the period of May, 21-29, 1989 (MJD.47668-47675), the residual error came to 0.11ms; what is more, from Jan.,20-25, 1990 (MJD.47911-47916), the residual is as high as $0.17 \mathrm{~ms}$ - about 6 times more than the average variance.

We checked all data and haven't found any mistake. Therefore, the error might be the result of irregular variation of the earth rotation. The problem is: what causes these abnormal variations? To seek the answer, we first use the same method to process the AAM series. Its residual is shown at the top of Figure 3. And there isn't distinct change in it. Hence, the abnormal variation of the earth rotation is probably not caused by AAM.

Laptukhov [2] has given such an opinion: A solar flare burst will bring about a shock wave. As soon as the shock wave reaches the magnetopause, the whole magnetosphere would be compressed. Then the horizontal component of magnetic field will increase about $20-100 \gamma$; it consequently causes a geomagnetic storm. When the outer magnetism field inducts the terrestrial current, it induces a magnetic field, in which the magnetic moment of the earth would be affected; as a result, the earth rotation rate might change. Though the opinion hasn't been confirmed by observation or by theory, we think it is an logical and reasonable assumption.

After consulting the data of strong solar flare burst and the index of geomagnetism, we notice that during the periods of March,8-16, 1989 and Nov.,7-17, 1989, there are a lot of very strong bursts of solar flares. The area of flares reached 1700 units. Shortly after, very strong geomagnetic storms occurred. There might be a time lag between the solar flare and the variation of LOD. In fact, the irregular variation of LOD didn't occur until 2 months later (the last 10 days of May,1989 and the middle days of Jan.,1990). Their corresponding relations are shown in Table 2. According to the precision of LOD, only those flares that burst strongly and with big apparent area can be reflected in the residual series of LOD. 
Table 2. Solar Flare Burst, Geomagnetic Storm and Abnormal Variation of LOD

\begin{tabular}{|c|c|c|c|}
\hline \multirow{4}{*}{ Flare } & occurring time & Mar. 8-16,89 & Nov.7-17,89 \\
\cline { 2 - 4 } & The biggest apparent area & 1763 & 1766 \\
\cline { 2 - 4 } & The highest class & $3 \mathrm{~B}$ & $3 \mathrm{~B}$ \\
\hline \multirow{2}{*}{$\begin{array}{c}\text { AP } \\
\text { Index }\end{array}$} & occurring time & Mar. 9-20,89 & Nov.13-19,89 \\
\cline { 2 - 4 } & the biggest magnitude & 98.2 & 64.3 \\
\hline \multirow{2}{*}{ LOD } & occurring time & May 21-29,89 & Jan.20-25,90 \\
\cline { 2 - 5 } & The biggest magnitude & $0.11 \mathrm{~ms}$ & $0.17 \mathrm{~ms}$ \\
\hline
\end{tabular}

\section{Conclusion}

Because the high precision of EOP given by the new technology (one order of magnitude higher than that given by the classical technology), our study on the rapid abnormal rotation of LOD become possible. There might be various reasons for the irregular rotation of LOD, but this might be an important mechanism, which means, when the solar flares burst strongly, the geomagnetic shell would be affected. It inducts electric current; affects the earth magnetic momentum, and leads to the irregular changes of LOD.

The research reported here is supported by the Nationa Natural Science Foundation of China.

\section{References}

1. Zheng D.W. and Dong D.N., Acta Astronomica SINICA,

Realization of Narrow Band Filtering of the Polar Motion Data

with Multi-stage Filter, Vol. 27, No.4, 368 (1986) (in Chinese)

2. Laptukhov.A.P., Geomagnetism and Aeronomy, Periodic Oscillation of Geomagnetic and Solar Activity, Vol. 22, 612-616 (1982) (in Russian) 


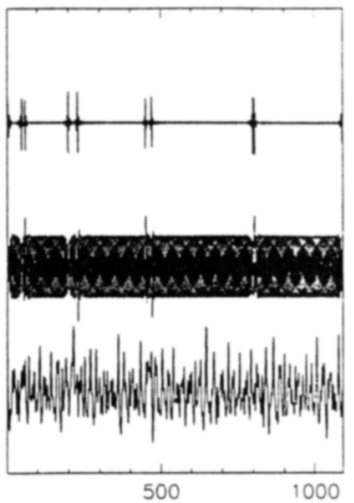

Fig 1 simuiated test
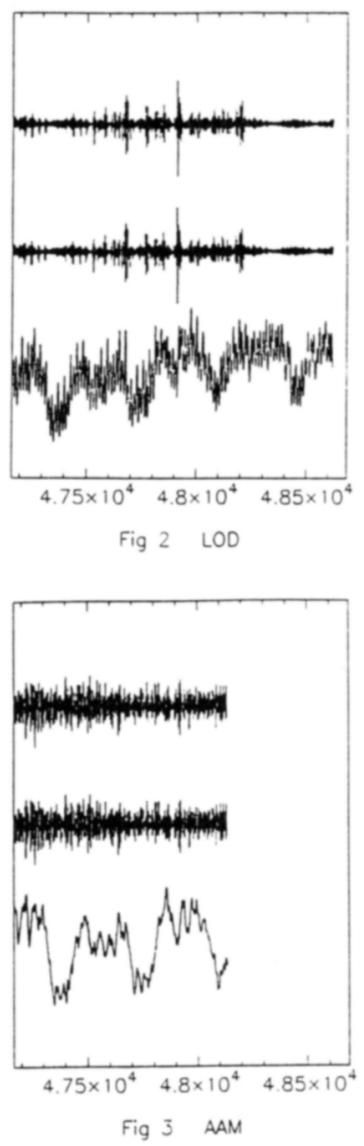